\title{
Nasal respiration entrains delta-frequency oscillations in the prefrontal cortex and hippocampus of rodents
}

\author{
Andre L. V. Lockmann ${ }^{1} \cdot$ Adriano B. L. Tort $^{1}$
}

Received: 7 July 2017 / Accepted: 10 November 2017 / Published online: 8 December 2017

c) Springer-Verlag GmbH Germany, part of Springer Nature 2017

\section{Dear Editor,}

We read with great interest the article "Prefrontal-hippocampal coupling by theta rhythm and $2-5 \mathrm{~Hz}$ oscillation in the delta band: The role of the nucleus reuniens of the thalamus" by Roy et al. (2017), published in Brain Structure and Function. The communication between the hippocampus and prefrontal cortex (PFC) is crucial for memory formation (Colgin 2011). Previous studies have shown that local field potentials (LFPs) in the PFC and hippocampus may synchronize in the theta-frequency range $(4-10 \mathrm{~Hz})$ (Jones and Wilson 2005; Sigurdsson et al. 2010; Benchenane et al. 2010). Since theta is generated in the hippocampus (Buzsáki 2002; Sirota et al. 2008), it is possible that these oscillations mediate information flow from this region to the PFC. Recording from urethane-anesthetized rats, Roy et al. (2017) have found a new $2-5 \mathrm{~Hz}$ oscillation and suggested that it mediates information transfer in the other direction, namely, from the PFC to the hippocampus, via a relay in the thalamic nucleus reuniens.

Interestingly, rodents breathe at the delta-frequency range ( 0.5-4 Hz) during anesthesia (Clement et al. 2008), therefore, at overlapping frequencies with the oscillations described by Roy et al. (2017). Rhythmic airflow is known to activate receptors in the nasal cavity and drive a prominent respiration-coupled LFP rhythm (RR) in olfactory brain areas such as the olfactory bulb and piriform cortex (Adrian 1942; Fontanini et al. 2003). The piriform cortex projects directly to the PFC (Clugnet and Price 1987) and indirectly to the hippocampus, after a relay in the entorhinal cortex (Wilson and Steward 1978).

We have recently characterized three different types of low-frequency oscillations $<6 \mathrm{~Hz}$ in LFPs from the olfactory

Andre L. V. Lockmann

andrelockmann@neuro.ufrn.br

1 Brain Institute, Federal University of Rio Grande do Norte, Natal, RN 59056-450, Brazil bulb, hippocampus, and PFC of urethane-anesthetized rats (Lockmann et al. 2016), the same experimental preparation as in Roy et al. (2017). By simultaneously assessing air pressure in the nasal cavity of these animals, we could demonstrate that one of the three oscillations actually corresponded to RR: it had the same frequency as and phase-locked to the breathing cycles (the other two oscillations corresponded to up-and-down state transitions and theta oscillations; Lockmann et al. 2016). We further showed that respirationentrained LFP oscillations were abolished by tracheostomy and restored by rhythmic air puffing into the nasal cavity; moreover, in the hippocampus, RR had the maximum amplitude in the dentate gyrus hilus, the anatomical site where olfactory inputs impinge (Lockmann et al. 2016).

Along with other recent papers in mice (Ito et al. 2014; Yanovsky et al. 2014; Nguyen Chi et al. 2016; Zhong et al. 2017; Biskamp et al. 2017; Tort et al. 2017), there is now solid evidence demonstrating the existence of respirationcoupled LFP oscillations in several regions of the rodent brain, not restricted to the olfactory areas. Importantly, RR is particularly prominent in frontal regions (Zhong et al. 2017; Biskamp et al. 2017; Tort et al. 2017). Roy et al. (2017) called the new rhythm as " $2-5 \mathrm{~Hz}$ oscillation" (as stated in the title), because its peak frequency increased with arousal induced by electrical stimulation of the reticular nucleus pontis oralis (RPO, their Fig. 3), though at the highest stimulation intensity, the peak frequency was $\sim 4 \mathrm{~Hz}$ (their Fig. 3d). Interestingly, the level of arousal also influences respiratory rate, and tail pinching has been previously shown to increase RR frequency (see examples in Yanovsky et al. 2014 and; Lockmann et al. 2016). In the absence of RPO stimulation, Roy et al. (2017) actually found an oscillation around $2 \mathrm{~Hz}$ (their Fig. 1), closer to the $\sim 1.5 \mathrm{~Hz}$ peak frequency of the RR reported in Lockmann et al. (2016). Moreover, as shown in their Fig. 6, hippocampal-prefrontal coherence also peaked at $\sim 2 \mathrm{~Hz}$. Of note, Roy et al. (2017) called "wide-band delta activity" what we and others have called the "slow oscillation" ( $\mathrm{SO}$ ) associated with 
a
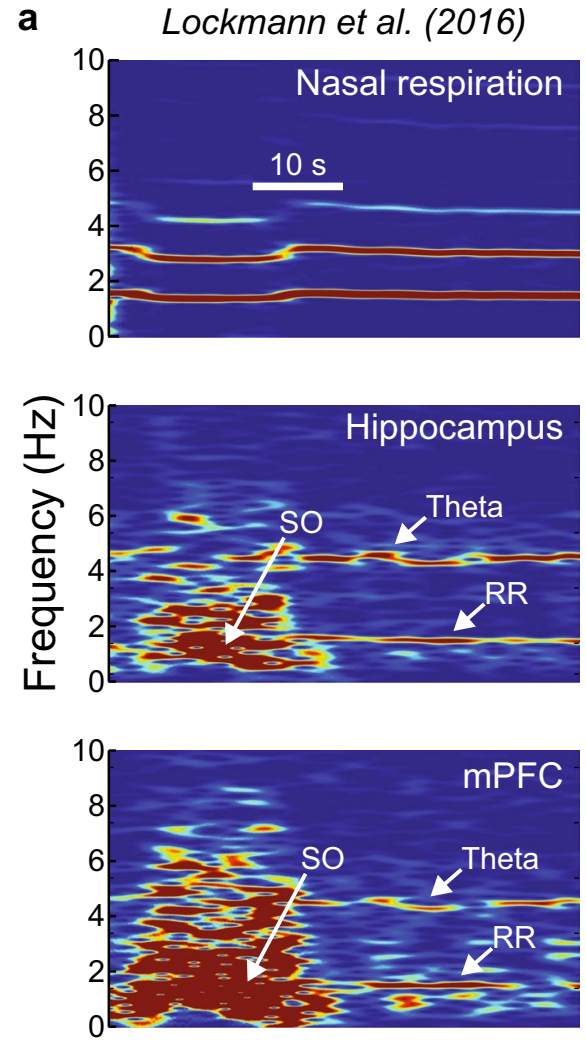

C

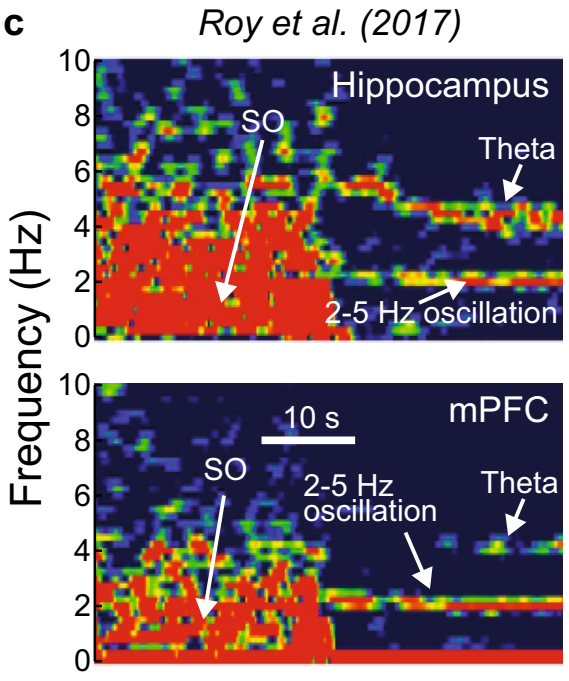

b SO period

Theta period
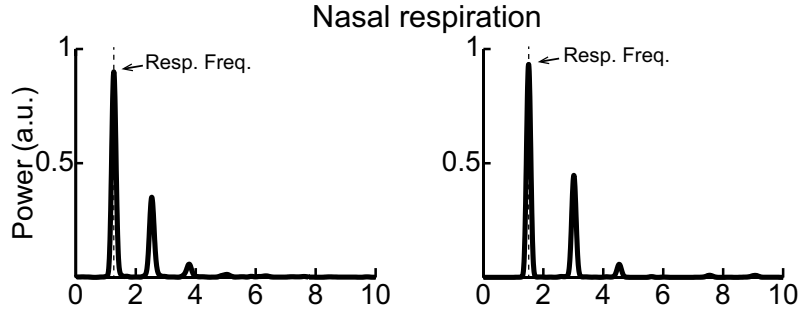

Hippocampus
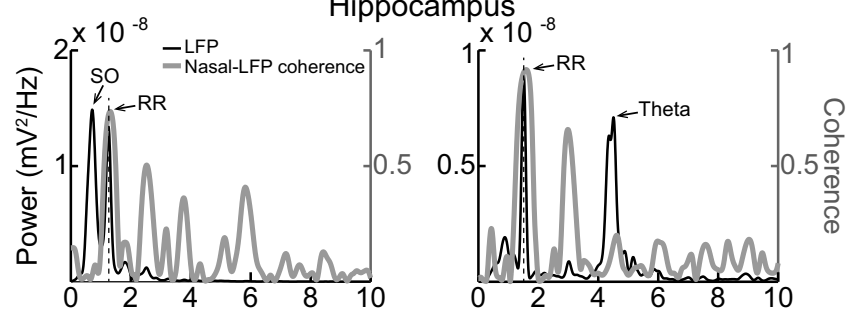

mPFC
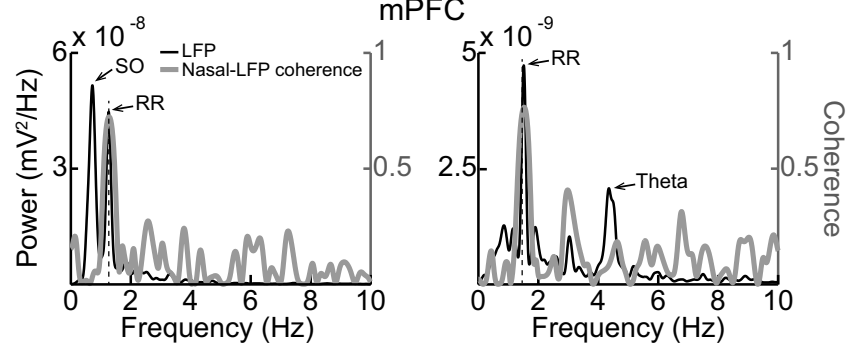

d
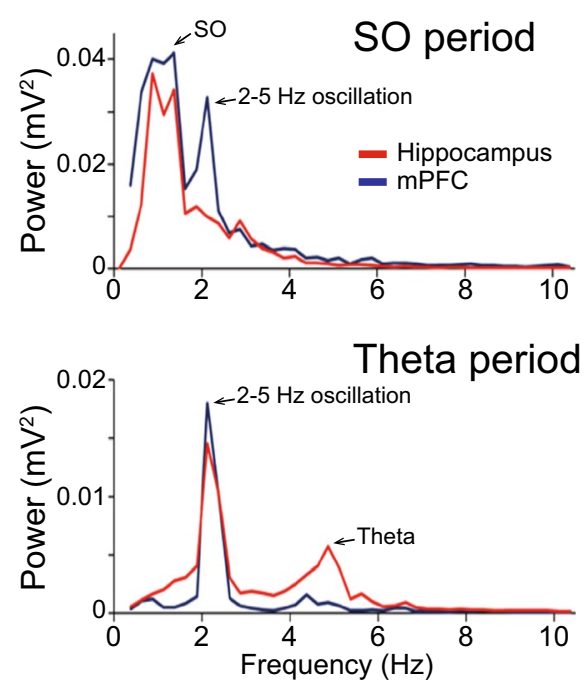

theta (right) periods. Gray traces depict coherence spectra between nasal respiration and LFP signals. Notice high coherence at the respiration frequency and its harmonics. c Time-frequency power analysis of LFP signals from the hippocampus (top) and mPFC (bottom) in a urethane-anesthetized rat during a similar SO-to-theta transition as in (a). d Power spectra of LFPs from the hippocampus (red) and mPFC (blue). Notice that the $2-5 \mathrm{~Hz}$ oscillation is strikingly similar to the RR shown in (a) and (b). a, b Computed de novo using data previously published in Lockmann et al. (2016). c, d Modified from Roy et al. (2017) 
up-and-down state transitions (Wolansky et al. 2006; Sharma et al. 2010; Viczko et al. 2014). During spontaneous thetaSO transitions, both the RR in Lockmann et al. (2016) and the $2-5 \mathrm{~Hz}$ oscillation in Roy et al. (2017) were faster than $\mathrm{SO}$ and slower than theta. We reproduce these findings in Fig. 1.

Although Roy et al. (2017) did not measure respiration in their experiments, the striking similarities with our findings (Fig. 1) suggest that the $2-5 \mathrm{~Hz}$ oscillation observed in the hippocampus and PFC by Roy et al. (2017) corresponds to RR, i.e., network activity entrained by nasal respiration. The recent boom of studies recording from these same brain regions has described RRs in both awake (Heck et al. 2016; Nguyen Chi et al. 2016; Tort et al. 2017; Zhong et al. 2017; Biskamp et al. 2017) and anesthetized (Yanovsky et al. 2014; Lockmann et al. 2016) rodents. In particular, Nguyen Chi et al. (2016) demonstrated that RR in head-fixed awake mice follows respiration rates from $2 \mathrm{~Hz}$ (immobility) to $10 \mathrm{~Hz}$ (running), while Biskamp et al. (2017), Zhong et al. (2017), and Tort et al. (2017) have all shown that RR is a prominent rhythm in the PFC of freely behaving mice. Altogether, these findings indicate that nasal respiration imposes low-frequency $(<10 \mathrm{~Hz})$ rhythmicity in several regions of the rodent brain, whose exact peak frequency depends on breathing rate.

In short, we congratulate Roy et al. (2017) for the very interesting work. Our intention with this letter is simply to call attention to the possibility that the $2-5 \mathrm{~Hz}$ oscillation reported by these authors would be the same as the respiration-entrained oscillations we and others have been describing. We particularly believe this is the case. At any event, these observations reinforce the importance of tracking nasal respiration along with electrophysiological recordings.

\section{Compliance with ethical standards}

Funding This study was funded by Conselho Nacional de Desenvolvimento Científico e Tecnológico and Coordenação de Aperfeiçoamento de Pessoal de Nível Superior.

\section{Conflict of interest The authors declare no conflict of interest.}

Ethical approval All applicable international, national, and institutional guidelines for the care and use of animals were followed. All procedures in animals were in accordance with the ethical standards of the institution at which the study was conducted.

\section{References}

Adrian ED (1942) Olfactory reactions in the brain of the hedgehog. J Physiol 100:459-473

Benchenane K, Peyrache A, Khamassi M et al (2010) Coherent theta oscillations and reorganization of spike timing in the hippocampal- prefrontal network upon learning. Neuron 66:921-936
Biskamp J, Bartos M, Sauer J-F (2017) Organization of prefrontal network activity by respiration-related oscillations. Sci Rep 7:45508

Buzsáki G (2002) Theta oscillations in the hippocampus. Neuron 33:325-340

Clement EA, Richard A, Thwaites M et al (2008) Cyclic and sleep-like spontaneous alternations of brain state under urethane anaesthesia. PLoS One 3:e2004

Clugnet M-C, Price JL (1987) Olfactory input to the prefrontal cortex in the rat. Ann N Y Acad Sci 510:231-235

Colgin LL (2011) Oscillations and hippocampal-prefrontal synchrony. Curr Opin Neurobiol 21:467-474

Fontanini A, Spano P, Bower JM (2003) Ketamine-xylazine-induced slow $(<1.5 \mathrm{~Hz})$ oscillations in the rat piriform (olfactory) cortex are functionally correlated with respiration. J Neurosci 23:7993-8001

Heck DH, McAfee SS, Liu Y et al (2016) Cortical rhythms are modulated by respiration. bioRxiv. https://doi.org/10.1101/049007

Ito J, Roy S, Liu Y et al (2014) Whisker barrel cortex delta oscillations and gamma power in the awake mouse are linked to respiration. Nat Commun 5:3572

Jones MW, Wilson MA (2005) Theta rhythms coordinate hippocampalprefrontal interactions in a spatial memory task. PLoS Biol 3:e402

Lockmann ALV, Laplagne DA, Leão RN, Tort ABL (2016) A respiration-coupled rhythm in the rat hippocampus independent of theta and slow oscillations. J Neurosci 36:5338-5352

Nguyen Chi V, Müller C, Wolfenstetter T et al (2016) Hippocampal respiration-driven rhythm distinct from theta oscillations in awake mice. J Neurosci 36:162-177

Roy A, Svensson FP, Mazeh A, Kocsis B (2017) Prefrontal-hippocampal coupling by theta rhythm and by $2-5 \mathrm{~Hz}$ oscillation in the delta band: the role of the nucleus reuniens of the thalamus. Brain Struct Funct 222:2819-2830. https://doi.org/10.1007/ s00429-017-1374-6

Sharma AV, Wolansky T, Dickson CT (2010) A comparison of sleeplike slow oscillations in the hippocampus under ketamine and urethane anesthesia. J Neurophysiol 104:932-939

Sigurdsson T, Stark KL, Karayiorgou M et al (2010) Impaired hippocampal-prefrontal synchrony in a genetic mouse model of schizophrenia. Nature 464:763-767

Sirota A, Montgomery S, Fujisawa S et al (2008) Entrainment of neocortical neurons and gamma oscillations by the hippocampal theta rhythm. Neuron 60:683-697

Tort ABL, Ponsel S, Jessberger J et al (2017) Parallel occurrence of theta and respiration-coupled network oscillations throughout the mouse brain. bioRxiv. https://doi.org/10.1101/139485

Viczko J, Sharma AV, Pagliardini S et al (2014) Lack of respiratory coupling with neocortical and hippocampal slow oscillations. J Neurosci 34:3937-3946

Wilson RC, Steward O (1978) Polysynaptic activation of the dentate gyrus of the hippocampal formation: an olfactory input via the lateral entorhinal cortex. Exp Brain Res 33:523-534

Wolansky T, Clement EA, Peters SR et al (2006) Hippocampal slow oscillation: a novel EEG state and its coordination with ongoing neocortical activity. J Neurosci 26:6213-6229

Yanovsky Y, Ciatipis M, Draguhn A et al (2014) Slow oscillations in the mouse hippocampus entrained by nasal respiration. J Neurosci 34:5949-5964

Zhong W, Ciatipis M, Wolfenstetter T et al (2017) Selective entrainment of gamma sub-bands by different slow network oscillations. Proc Natl Acad Sci USA 114:4519-4524 